\title{
Response of the polaron system consisting of an impurity in a Bose-Einstein condensate to Bragg spectroscopy
}

\author{
W. Casteels, ${ }^{1}$ J. Tempere, ${ }^{1,2}$ and J. T. Devreese ${ }^{1}$ \\ ${ }^{1}$ TQC, Universiteit Antwerpen, Groenenborgerlaan 171, B-2020 Antwerpen, Belgium \\ ${ }^{2}$ Lyman Laboratory of Physics, Harvard University, Cambridge, Massachusetts 02138, USA
}

(Received 30 September 2010; published 31 March 2011)

\begin{abstract}
We expand the existing polaron response theory, expressed within the Mori-Zwanzig projection operator formalism applicable for the transfer of arbitrary energy and zero momentum, for the case of finite momentum exchange. A general formula is then derived that can be used to calculate the response of a system to a probe that transfers both momentum and energy to the system. The main extension of the existing polaron response theory is the finite momentum exchange, which was not needed until now, since it is negligible for optical absorption. However, this formalism is needed to calculate the response of the polaronic system consisting of an impurity in a Bose-Einstein condensate (BEC) to Bragg spectroscopy. We show that the well-known features that appear in the optical absorption of the solid-state Fröhlich polaron are also present in the Bragg response of the BEC-impurity polaron. The f-sum rule is written in a form suitable to provide an independent consistency test for our results. The effect of lifetime broadening on the BEC-impurity spectrum is examined. The results derived here are discussed in the framework of an experimental realization consisting of a lithium impurity in a sodium condensate.
\end{abstract}

DOI: 10.1103/PhysRevA.83.033631

PACS number(s): 03.75.Nt, 71.38.Fp, 03.75.Hh

\section{INTRODUCTION}

The experimental realization of impurities in a BoseEinstein condensate (BEC) [1-3] and the possibility to produce quantum degenerate atomic mixtures [4-6] has resulted in an interest in the physics of impurities in a quantum gas. This has led to an investigation of the change of the properties of the bare impurities as a result of the interactions with the condensate (such as the effective interaction [7,8] and the effective mass [9-11]) and a study of the self-trapping of the impurity [12,13]. Also, the system of a spin-down fermion in a sea of spin-up fermions, usually indicated as the Fermi polaron, has been investigated, which resulted in good agreement between theory $[14,15]$ and experiments $[16,17]$. Furthermore, it was shown that, when the Bogoliubov approximation is valid, the system of an impurity in a BEC can be mapped to the Fröhlich polaron system [18-20], which shows that this system can be added to the list of condensed-matter systems that can be imitated in the context of quantum gases [21]. The experimental realization of quantum degenerate atomic mixtures in an optical lattice [22-24] has led to the theoretical study of the polaronic properties of impurities in a BEC where the influence of the optical lattice is felt only by the impurities $[25,26]$ or by both the impurities and the condensate [27].

In the context of solid-state physics, the Fröhlich polaron consists of a charge carrier (electron, hole) interacting with the phonons in an ionic crystal or a polar semiconductor [28,29]. This Fröhlich polaron Hamiltonian cannot be diagonalized exactly, and several approximation methods have been developed for it. The most general way to study the system is through a variational principle within the path-integral formalism, which was developed by Feynman [30]. The internal excitation spectrum of the Fröhlich polaron was revealed through the calculation of the optical absorption by Devreese et al. [31], based on the Feynman path-integral method and a response formalism introduced in Ref. [32]. Recently, the excitation spectrum of the Fröhlich polaron Hamiltonian was also studied numerically with diagrammatic quantum Monte Carlo numerical techniques, and these results showed deviations in the large coupling optical absorption linewidth with the theoretical results of Ref. [31], see Ref. [33]. At weak and intermediate couplings, the analytical theory of Ref. [31] agrees well with the numerical simulations of Ref. [33], and this optical absorption spectrum was also studied experimentally [34]. So far, the strong-coupling regime could not be probed experimentally since the largest attainable Fröhlich polaron coupling constant in any solid is not large enough. The large coupling behavior remains an important question since a better understanding of the intermediate- and strong-coupling regimes might be useful to elucidate the role of polarons and bipolarons in unconventional pairing mechanisms, e.g., for high-temperature superconductivity [35]. Furthermore, it was shown that, at intermediate coupling, the introduction of an ad hoc lifetime $\tau$ for the eigenstates of the polaron model system is an improvement of the theory that is called the extended memory function formalism [36].

If the Bogoliubov approximation is applicable, the Hamiltonian of an impurity in a BEC can be written as the sum of the mean field energy of the homogeneous condensate and the Fröhlich polaron Hamiltonian that describes the fluctuations [20]:

$$
\widehat{H}_{\mathrm{pol}}=\frac{\widehat{p}^{2}}{2 m_{I}}+\sum_{\vec{k} \neq 0} \hbar \omega_{\vec{k}} \widehat{b}_{\vec{k}}^{\dagger} \widehat{b}_{\vec{k}}+\sum_{\vec{k} \neq 0} V_{\vec{k}} e^{i \vec{k} \cdot \widehat{r}}\left(\widehat{b}_{\vec{k}}+\widehat{b}_{-\vec{k}}^{\dagger}\right),
$$

which describes the interaction between the impurity and the Bogoliubov excitations where $\widehat{r}$ and $\widehat{p}$ represent the position and momentum of the impurity with mass $m_{I}, \widehat{b}_{\vec{k}}$ and $\widehat{b}_{\vec{k}}^{\dagger}$ are the annihilation and creation operators for the Bogoliubov excitations, $\omega_{\vec{k}}$ is the Bogoliubov frequency,

$$
\omega_{\vec{k}}=c k \sqrt{1+(\xi k)^{2} / 2},
$$


and $V_{\vec{k}}$ is the interaction amplitude,

$$
V_{\vec{k}}=\sqrt{N_{0}}\left[\frac{(\xi k)^{2}}{(\xi k)^{2}+2}\right]^{1 / 4} g_{\mathrm{IB}} .
$$

In the above expressions, use was made of the definition of the healing length of the condensate $\xi=1 / \sqrt{8 \pi a_{\mathrm{BB}} n_{0}}$ with $n_{0}=N_{0} / V$ as the condensate density and $a_{\mathrm{BB}}$ as the boson-boson scattering length. We also used the expression for the speed of sound in the condensate $c=\hbar /\left(\sqrt{2} m_{B} \xi\right)$. In Ref. [20], the Feynman variational path-integral technique was applied to this Hamiltonian, and an upper bound for the free energy was calculated by using the Jensen-Feynman inequality with the Feynman model system. This model system consists of the impurity mass coupled to another mass $M$ by a spring with frequency $\Omega$. The parameters $M$ and $\Omega$ are then used to minimize the free energy. It followed that the Fröhlich polaron coupling parameter in this case is given by

$$
\alpha=\frac{a_{\mathrm{IB}}^{2}}{a_{\mathrm{BB}} \xi},
$$

with $a_{\mathrm{IB}}$ as the impurity-boson scattering length. Together with the ratio between the masses of the impurity and the bosons, this coupling parameter fully determines the static properties of the specific BEC-impurity polaron system. Depending on the value of this coupling strength, two regimes were identified: a strong-coupling regime, which has properties that suggested a polaronic self-trapped state and a weak-coupling regime, which suggested a free polaron. Since this coupling parameter depends strongly on the scattering lengths, which can be tuned externally by a magnetic field through a Feshbach resonance (see, e.g., Ref. [37]), it may be possible to tune the system experimentally to the regime of strong coupling. This technique might enable the experimental realization of the strong-coupling regime and reveal the internal structure of the Fröhlich polaron Hamiltonian.

Since, as opposed to the original Fröhlich polaron in solid state, the BEC-impurity polaron system is not charged, it is not helpful to perform optical absorption measurements on this system. The method to probe the system is addressed in the present paper and will be shown to be Bragg spectroscopy. This has proven to be a very successful tool to probe the structure of BECs (see, e.g., Refs. [38] and [39]). It is realized by probing the system with two laser beams with wave vectors $\vec{k}_{1}$ and $\vec{k}_{2}$ and frequencies $\omega_{1}$ and $\omega_{2}$. An atom can then undergo a stimulated scattering event by absorbing a photon from beam 1 and emitting it in beam 2, which changes its momentum by $\vec{k}=\vec{k}_{1}-\vec{k}_{2}$ and its energy by $\hbar \omega=\hbar \omega_{1}-\hbar \omega_{2}$. See Fig. 1 for a schematical picture of a typical experimental setup. A possible way to measure the response of the system is by performing a time-of-flight experiment and count the number of atoms $N_{\text {Bragg }}$ that have gained a momentum $\vec{k}$. Within the formalism of linear-response theory, this number is given by [37]

$$
N_{\text {Bragg }}=\frac{2}{\hbar}\left(\frac{V}{2}\right)^{2} \tau \operatorname{Im} \chi(\omega, \vec{k}),
$$

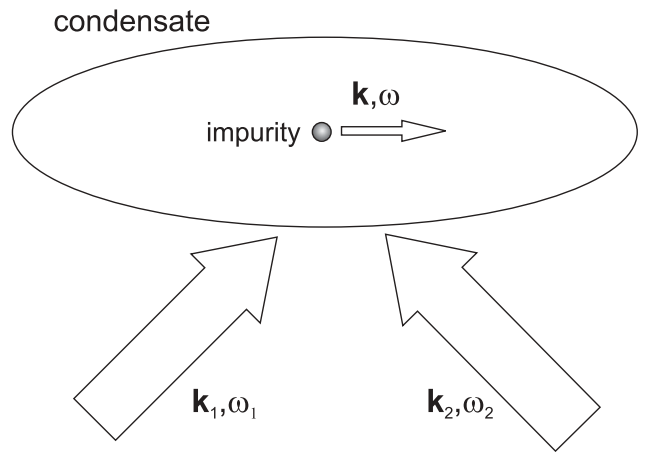

FIG. 1. Schematical picture of a typical experimental setup for Bragg spectroscopy. Two lasers with momentum $\vec{k}_{1}$ and $\vec{k}_{2}$ and energy $\hbar \omega_{1}$ and $\hbar \omega_{2}$ are impinged upon the impurity in the condensate that gains an energy $\hbar \omega=\hbar \omega_{1}-\hbar \omega_{2}$ and a momentum $\vec{k}=\vec{k}_{1}-\vec{k}_{2}$.

with $\tau$ as the duration of the pulse, $V$ as the amplitude of the laser-induced potential, and $\chi(\omega, \vec{k})$ as the density response function, defined by

$$
\begin{aligned}
\chi(\omega, \vec{k})= & -\frac{1}{\hbar} \mathcal{Z}^{-1} \sum_{m, n} e^{-\beta E_{m}} \\
& \times\left[\frac{\left|\left\langle m\left|\rho_{\vec{k}}\right| n\right\rangle\right|^{2}}{\omega-\omega_{n m}+i \delta}-\frac{\left|\left\langle m\left|\rho_{\vec{k}}^{\dagger}\right| n\right\rangle\right|^{2}}{\omega+\omega_{n m}+i \delta}\right],
\end{aligned}
$$

with $\rho_{\vec{k}}$ as the induced density of the impurity, $\mathcal{Z}$ as the partition function, $|n\rangle$ as the eigenstates of the unperturbed system, and $\omega_{n m}=\left(E_{n}-E_{m}\right) / \hbar$ as the transition frequencies. This density response function can be rewritten as the Fourier transform of the retarded density-density correlation function:

$$
\chi(\omega, \vec{k})=\frac{i}{\hbar} \int_{0}^{\infty} d t e^{i \omega t}\left\langle\left[\rho_{\vec{k}}(t), \rho_{\vec{k}}^{\dagger}\right]\right\rangle .
$$

The retarded density-density correlation function is needed to obtain the response of a system to Bragg spectroscopy.

We will start by using the Mori formalism to calculate a general expression for the density-density correlation function (7) and then will apply it to the generic polaron system with arbitrary dispersion and interaction amplitude. Then, we show that, if we calculate the current-current correlation for the $\vec{k}=0$ limit from this expression, we find the known result for the optical absorption of Ref. [31]. Next, we introduce the dispersion and interaction amplitude for the BEC-impurity polaron and obtain results for the response to Bragg spectroscopy. The result is expressed as a double integral over highly oscillatory integrands that has to be calculated numerically and which can give numerical problems. To overcome these problems, then, we will rewrite our expressions in another representation that is suitable for numerical work. We then write down the f-sum rule to check our results and find that, at low temperatures, there is a relatively large recoil contribution for $\omega \rightarrow 0$, which numerically has to be added separately in the sum rule. This behavior was also found in the optical absorption of the solid-state Fröhlich polaron, and, in that case, there is a relation with the effective mass of the polaron [40]. Recently, this sum rule was used to experimentally determine the effective mass of the solid-state Fröhlich polaron, see Refs. [41] and [42]. Next, following De Filippis et al. [36], we introduce a lifetime $\tau$ and examine the dependence of 
the theory on this lifetime. The results are analyzed for the experimental setup consisting of a lithium impurity in a sodium condensate, which is the same system that was examined in Ref. [20].

\section{APPLICATION OF THE MORI FORMALISM TO THE DENSITY-DENSITY CORRELATION}

The Mori-Zwanzig projection operator technique (see Refs. [43] and [44]) is a well-established technique to calculate correlation functions and has been applied before to the Fröhlich polaron Hamiltonian to calculate the optical absorption by Peeters and Devreese [45] (for a detailed description, we refer to Ref. [46]). This technique can also be applied to the calculation of the density-density correlation function. However, there is one obstacle that we encountered when defining the Mori projection operator. The reason is that the common definition of this operator is ill defined since it leads to a vanishing density-density commutator. This problem was already noticed by Ichiyanagi [47], and he suggested the following definition for the action of the Mori projection operator $P$ on an arbitrary operator $A$ :

$$
P A=\frac{\left\langle\left[A, \dot{\rho}_{\vec{k}}^{\dagger}\right]\right\rangle}{\left\langle\left[\rho_{\vec{k}}, \dot{\rho}_{\vec{k}}^{\dagger}\right]\right\rangle} \rho_{\vec{k}} .
$$

In our calculations, we have followed this suggestion and have found that the density response function can be written as

$$
\chi(\omega, \vec{k})=\frac{i}{\hbar} \frac{\varphi}{\omega^{2}+O-\Sigma(\omega, \vec{k})},
$$

with

$$
\begin{gathered}
\varphi=\left\langle\left[\rho_{\vec{k}}, \dot{\rho}_{\vec{k}}^{\dagger}\right]\right\rangle, \\
O=\frac{\omega}{\varphi}\left\langle\left[L \rho_{\vec{k}}, \dot{\rho}_{\vec{k}}^{\dagger}\right]\right\rangle, \\
\Sigma(\omega, \vec{k})=-\frac{1}{\varphi} \int_{0}^{\infty} d t\left(1-e^{i \omega t}\right)\left\langle\left[\mathcal{B}_{\vec{k}}(t), \mathcal{B}_{\vec{k}}^{\dagger}\right]\right\rangle,
\end{gathered}
$$

where $L$ is the Liouville operator and $\Sigma(\omega, \vec{k})$ is known as the memory function or self-energy. Furthermore, we also introduced the operator $\mathcal{B}_{\vec{k}}(t)$ defined as

$$
\mathcal{B}_{\vec{k}}=Q L \dot{\rho}_{\vec{k}}
$$

with $Q$ as the complementary Mori projection operator $Q=1-P$. The time dependence of $\mathcal{B}_{\vec{k}}(t)$ is governed by a new Liouville operator defined by $\mathcal{L}=Q L Q$. Here, it is important to note that, until this point, no approximations were made, and the result (9) for the linear density response function is exact. Also, to derive the formula (9), we have not introduced a specific system, and so, it is a general result for the density response function and can also be applied to calculate the response of other systems to probes with an arbitrary energy and momentum exchange. For example, the final state of neutron scattering is also determined by the density response function [48]. We now consider the Fröhlich polaron Hamiltonian and follow an approach similar to that used in Ref. [45]. This consists of replacing the new Liouville operator $\mathcal{L}$ by the sum of the Liouville operator of free Bogoliubov excitations $L_{\mathrm{Bog}}$ and the Liouville operator of the Feynman model system $L_{F}: \mathcal{L} \rightarrow L_{\text {Bog }}+L_{F}$. Within this approximation, we can calculate the different quantities in the expression (9), which gives $O=0$ and

$$
\varphi=i \frac{\hbar k^{2}}{m_{I}} N,
$$

where $N$ is the number of impurities. The memory function is then given by

$$
\begin{aligned}
\Sigma(\omega, \vec{k})= & \frac{2}{m_{I} N \hbar} \sum_{\vec{q} \neq 0}\left|V_{\vec{q}}\right|^{2} \frac{(\vec{k} \cdot \vec{q})^{2}}{k^{2}} \int_{0}^{\infty} d t\left(1-e^{i \omega t}\right) \\
& \times \operatorname{Im}\left\{\left[e^{i \omega_{\vec{q}} t}+2 \cos \left(\omega_{\vec{q}} t\right) n_{\vec{q}}\right] \exp \left[-(\vec{k}+\vec{q})^{2} D(t)\right]\right\},
\end{aligned}
$$

with $n_{\vec{q}}$ as the Bose-Einstein distribution, i.e., the number of Bogoliubov excitations with frequency $\omega_{\vec{q}}$ and $D(t)$ describes the Feynman model system and is given by

$$
\begin{aligned}
D(t)= & \frac{t^{2}}{2 \beta(m+M)}-i \frac{\hbar}{2(m+M)} t+\frac{\hbar M}{2 m \Omega(m+M)} \\
& \times\left[1-\exp (i \Omega t)+4 \sin ^{2}\left(\frac{\Omega t}{2}\right) n(\Omega)\right],
\end{aligned}
$$

with $M$ and $\Omega$ as the variational parameters. The values of these parameters are deduced from the minimalization of the free energy [20].

\section{LINK WITH THE OPTICAL ABSORPTION OF THE SOLID-STATE FRÖHLICH POLARON}

A well-known result from linear-response theory and the Kubo formalism is that the optical absorption of a system can be expressed as a current-current correlation function [49]. Since the current and the density are related to each other through the continuity equation, it is possible to calculate the current-current correlation function from the density-density correlation function. For the process of optical absorption, the photon momentum is negligible in comparison to the other momenta involved in the polaron problem, which is not the case for Bragg spectroscopy. To obtain the optical absorption, we have to take the limit $\vec{k} \rightarrow 0$, which gives, for the memory function,

$$
\begin{aligned}
\Sigma(\omega, 0)= & \frac{2}{3 m_{I} N \hbar} \sum_{\vec{q} \neq 0}\left|V_{\vec{q}}\right|^{2} q^{2} \int_{0}^{\infty} d t\left(1-e^{i \omega t}\right) \\
& \times \operatorname{Im}\left\{\left[e^{i \omega_{\vec{q}} t}+2 \cos \left(\omega_{\vec{q}} t\right) n_{\vec{q}}\right] \exp \left[-q^{2} D(t)\right]\right\} .
\end{aligned}
$$

This is just the memory function $\Sigma(\omega)$ calculated in Ref. [45] multiplied by $\omega$ :

$$
\Sigma(\omega, 0)=\omega \Sigma(\omega) .
$$

For the solid-state Fröhlich polaron, it is the current-current correlation that is needed for the optical absorption. By applying two partial integrations and making use of the continuity equation, one can find a simple relation between the two:

$$
\chi(\omega, \vec{k})=-\frac{i}{\hbar \omega^{2}} \int_{0}^{\infty} d t e^{i \omega t}\left\langle\left[\vec{k} \cdot \vec{j}_{\vec{k}}(t), \vec{k} \cdot \vec{j}_{\vec{k}}^{\dagger}\right]\right\rangle
$$


We now make use of the standard Kubo formula for the optical conductivity (see, e.g., Ref. [49]):

$$
\begin{aligned}
\operatorname{Re}[\sigma(\omega)] & =\operatorname{Re}\left[i \frac{e^{2}}{V m \omega}+\frac{1}{\hbar \omega} \int_{0}^{\infty} d t e^{i \omega t}\left\langle\left[j_{x}(t), j_{x}\right]\right\rangle\right] \\
& =\lim _{\vec{k} \rightarrow 0} \operatorname{Im}\left[-\frac{\hbar \omega}{k^{2}} \chi(\omega, \vec{k})\right] \\
& =\operatorname{Im}\left[-\frac{1}{m_{I}} \frac{1}{\omega-\Sigma(\omega)}\right] .
\end{aligned}
$$

This is the result that was found in Ref. [31] from the Feynman path-integral formalism and rederived in Ref. [45] using Mori's formalism.

\section{RESULTS FOR THE BEC-IMPURITY POLARON}

In this section, we will introduce the dispersion and the interaction amplitude for the BEC-impurity system and will obtain an expression for the response of the system to a Bragg pulse. From expression (5), it follows that the response of the system is characterized by the imaginary part of the density response function, which can be written as

$$
\begin{aligned}
& \operatorname{Im}[\chi(\omega, \vec{k})] \\
& \quad=-\frac{k^{2}}{m_{I}} N \frac{\operatorname{Im}[\Sigma(\omega, \vec{k})]}{\left\{\omega^{2}-\operatorname{Re}[\Sigma(\omega, \vec{k})]\right\}^{2}+\{\operatorname{Im}[\Sigma(\omega, \vec{k})]\}^{2}}
\end{aligned}
$$

This particular form for the response function is general for the Fröhlich polaron and was first introduced in Ref. [31]. The memory function for the BEC-impurity system is given by (with polaronic units, i.e., $\hbar=m_{I}=\xi=1$ )

$$
\begin{aligned}
\Sigma(\omega, \vec{k})= & \frac{1}{N} \frac{\alpha}{4 \pi}\left(\frac{m_{B}+1}{m_{B}}\right)^{2} \int_{0}^{\infty} d q \frac{q^{5}}{\sqrt{q+2}} \\
& \times \int_{0}^{\infty} d \widetilde{t}\left(1-e^{i \omega t}\right) \operatorname{Im}\left[\frac{\left[e^{i \omega_{\vec{q}} t}+2 \cos \left(\omega_{\vec{q}} t\right) n_{\vec{q}}\right]}{[2 k q D(t)]^{3}}\right. \\
& \times\left(\exp \left[-(k-q)^{2} D(t)\right]\left\{[1-2 k q D(t)]^{2}+1\right\}\right. \\
& \left.\left.-\exp \left[-(k+q)^{2} D(t)\right]\left\{[1+2 k q D(t)]^{2}+1\right\}\right)\right] .
\end{aligned}
$$

The double integral in expression (22) could not be calculated analytically, and it was computed numerically.

\section{SECOND REPRESENTATION FOR THE IMAGINARY PART OF THE MEMORY FUNCTION}

Due to the highly oscillatory behavior of the integrand, it turns out that expression (22) is not of a form efficient for numerical calculation of the imaginary part of the memory function. Therefore, we develop an alternative representation for the memory function that allows performing the time integration analytically. This provides a representation for the imaginary part that is well suited for numerical calculations. The real part of the memory function turns out to be far more involved, but it seems that, in this case, we can use expression (22). We will follow a similar approach as used in Ref. [31] and also in Ref. [45]. We start by rewriting Eq. (16) as

$$
\begin{aligned}
D(t)= & \frac{t^{2}}{2 \beta(m+M)}-i \frac{\hbar}{2(m+M)} t+\frac{\hbar M}{2 m \Omega(m+M)} \\
& \times\left[\operatorname{coth}\left(\frac{\hbar \beta \Omega}{2}\right)-[1+n(\Omega)] \exp (i \Omega t)\right. \\
& -n(\Omega) \exp (-i \Omega t)] .
\end{aligned}
$$

After two Taylor expansions for the two exponentials and writing the term with $t^{2}$ as a Gaussian integral, we obtain

$$
\begin{aligned}
\exp \left[-(\vec{k}+\vec{q})^{2} D(t)\right]= & \left(\frac{\beta}{2 \pi(m+M)}\right)^{1 / 2} \exp \left[-(\vec{k}+\vec{q})^{2} \frac{\hbar M}{2 m \Omega(m+M)} \operatorname{coth}\left(\frac{\hbar \beta \Omega}{2}\right)\right] \\
& \times \sum_{n, n^{\prime}}^{\infty} \frac{1}{n !} \frac{1}{n^{\prime} !}\left[\frac{\hbar M}{2 m \Omega(m+M)}[1+n(\Omega)]\right]^{n}\left[\frac{\hbar M}{2 m \Omega(m+M)} n(\Omega)\right]^{n^{\prime}}(\vec{k}+\vec{q})^{2\left(n+n^{\prime}\right)} \\
& \times \int_{-\infty}^{\infty} d p \exp \left[-\frac{\beta p^{2}}{2(M+m)}\right] \exp \left\{i t\left[\frac{\hbar(\vec{k}+\vec{q})^{2}}{2(m+M)} \pm \frac{p|\vec{k}+\vec{q}|}{m+M}+\left(n-n^{\prime}\right) \Omega\right]\right\}
\end{aligned}
$$

Furthermore, the memory function can be written as

$$
\begin{aligned}
\Sigma(\omega, \vec{k}) & =\frac{\hbar \omega}{\chi} \int_{0}^{\infty} d t e^{i \omega t} \int_{0}^{\beta} d \lambda\left\langle\mathcal{B}_{\vec{k}}(t-i \hbar \lambda) \mathcal{B}_{\vec{k}}^{\dagger}\right\rangle \\
& =-\frac{i \omega}{N m} \sum_{\vec{q} \neq 0}\left|V_{\vec{q}}\right|^{2} \frac{(\vec{k} \cdot \vec{q})^{2}}{k^{2}} \int_{0}^{\infty} d t e^{i \omega t} \int_{0}^{\beta} d \lambda\left[\left(1+n_{\vec{q}}\right) e^{-i \omega_{\vec{q}}(t-i \hbar \lambda)}+n_{\vec{q}} e^{i \omega_{\vec{q}}(t-i \hbar \lambda)}\right] \exp \left[-(\vec{k}+\vec{q})^{2} D(-t+i \hbar \lambda)\right]
\end{aligned}
$$


Now, the time integration is straightforward,

$$
\begin{aligned}
\Sigma(\omega, \vec{k})= & \frac{\omega}{N m}\left(\frac{\beta}{2 \pi(m+M)}\right)^{1 / 2} \sum_{\vec{q} \neq 0}\left|V_{\vec{q}}\right|^{2} e^{-(\vec{k}+\vec{q})^{2} a^{2}(\beta)} \frac{(\vec{k} \cdot \vec{q})^{2}}{k^{2}} \sum_{n, n^{\prime}}^{\infty} B\left(\beta, n, n^{\prime}\right)(\vec{k}+\vec{q})^{2\left(n+n^{\prime}\right)} \\
& \times \int_{-\infty}^{\infty} d p \exp \left[-\frac{\beta p^{2}}{2(M+m)}\right]\left[\frac{1-e^{-\hbar \beta \nu_{-}}}{\hbar v_{-}} \frac{n_{\vec{q}}}{\omega-v_{-}+i \varepsilon}+\frac{1-e^{-\hbar \beta v_{+}}}{\hbar v_{+}} \frac{1+n_{\vec{q}}}{\omega-v_{+}+i \varepsilon}\right],
\end{aligned}
$$

with the following notation:

$$
\begin{aligned}
a^{2}(\beta) & =\frac{\hbar M}{2 m \Omega(m+M)} \operatorname{coth}\left(\frac{\hbar \beta \Omega}{2}\right), \\
B\left(\beta, n, n^{\prime}\right) & =\frac{1}{n !} \frac{1}{n^{\prime} !}\left\{a^{2}[1+n(\Omega)]\right\}^{n}\left[a^{2} n(\Omega)\right]^{n^{\prime}},
\end{aligned}
$$

$$
\nu_{ \pm}= \pm \omega_{\vec{q}}+\frac{\hbar(\vec{k}+\vec{q})^{2}}{2(m+M)} \pm \frac{p|\vec{k}+\vec{q}|}{m+M}+\left(n-n^{\prime}\right) \Omega .
$$

The memory function is now split in an imaginary and a real part with the formula of Plemelj. The integral over $p$ in the imaginary part of Eq. (26) can be performed easily by using the $\delta$ function. Taking the dispersion and the interaction amplitude into account, this yields (with polaronic units)

$$
\begin{aligned}
\operatorname{Im}[\Sigma(\omega, \vec{k})]= & -\frac{1}{N} \frac{\alpha}{8}\left(\frac{m_{B}+1}{m_{B}}\right)^{2}\left(\frac{\beta(1+M)}{2 \pi}\right)^{1 / 2} \int_{0}^{\infty} d q q^{2} \int_{-1}^{1} d x \sqrt{\frac{q^{2}}{q^{2}+2}} e^{-\left(k^{2}+q^{2}+2 q k x\right) a^{2}(\beta)}(q x)^{2} \\
& \times \sum_{n, n^{\prime}}^{\infty} B\left(\beta, n, n^{\prime}\right)\left(k^{2}+q^{2}+2 q k x\right)^{\left(n+n^{\prime}\right)-1 / 2}\left(1-e^{-\beta \omega}\right) \\
& \times\left[n_{\vec{q}} \exp \left\{-\frac{\beta(M+1) A_{n}^{-}(\omega)^{2}}{2\left(k^{2}+q^{2}+2 q k x\right)}\right\}+\left(1+n_{\vec{q}}\right) \exp \left\{-\frac{\beta(M+1) A_{n}^{+}(\omega)^{2}}{2\left(k^{2}+q^{2}+2 q k x\right)}\right\}\right]
\end{aligned}
$$

with

$$
A_{n}^{ \pm}(\omega)= \pm \omega_{\vec{q}}+\frac{(\vec{k}+\vec{q})^{2}}{2(1+M)}+\left(n-n^{\prime}\right) \Omega-\omega
$$

As shown in Ref. [45], this expression has a very natural interpretation: Every term in the double sum corresponds to a well-defined physical process. The $\left(n, n^{\prime}\right)$ th term in the summation represents a scattering process of the polaron where, in the initial state, the polaron is in the $n^{\prime}$ th internal Franck-Condon (FC) state of the Feynman polaron model system and is then scattered to the $n$th internal FC state with the absorption of an energy $\hbar \omega$ and a momentum $\vec{k}$ through the Bragg scattering and with the emission (or absorption) of a Bogoliubov excitation with energy $\hbar \omega_{\vec{q}}$.

\section{SUM RULE}

As a consistency check of our results, we can apply the f-sum rule to our results, this can be written as

$$
\int_{0}^{\infty} d \omega \omega \operatorname{Im}[\chi(\omega, \vec{k})]=N \frac{\pi}{2} \frac{k^{2}}{m_{I}} .
$$

At small $\omega$ and as temperature tends to zero, a $\delta$ peak appears in the integrand for which, in numerical calculations, we have to add the contribution separately. This feature was found for the solid-state Fröhlich polaron, where the contribution of the $\delta$ peak can be used to determine the effective mass of the polaron [40]. The $\delta$ peak can be revealed if we look at the $\omega \rightarrow 0$ limits of the imaginary and real parts of expression (22) for the memory function,

$$
\begin{aligned}
& \lim _{\omega \rightarrow 0} \operatorname{Im}[\Sigma(\omega, \vec{k})]=0, \\
& \lim _{\omega \rightarrow 0} \frac{\operatorname{Re}[\Sigma(\omega, \vec{k})]}{\omega^{2}}=R(\alpha, \vec{k}),
\end{aligned}
$$


with

$$
\begin{aligned}
R(\alpha, \vec{k})= & \frac{\omega_{0}^{2}}{N} \frac{\alpha}{4 \pi}\left(\frac{m_{B}+1}{m_{B}}\right)^{2} \int_{0}^{\infty} d \widetilde{q} \widetilde{q}^{2} \frac{q}{\sqrt{q+2}} q^{2} \int_{0}^{\infty} d t \frac{t^{2}}{2} \operatorname{Im}\left[\frac { [ e ^ { i \omega _ { \vec { q } } t } + 2 \operatorname { c o s } ( \omega _ { \vec { q } } t ) n _ { \vec { q } } ] } { [ 2 k q D ( t ) ] ^ { 3 } } \left(\exp \left[-(k-q)^{2} D(t)\right]\right.\right. \\
& \left.\left.\times\left\{[1-2 k q D(t)]^{2}+1\right\}-\exp \left[-(k+q)^{2} D(t)\right]\left\{[1+2 k q D(t)]^{2}+1\right\}\right)\right]
\end{aligned}
$$

Now, if we look at the $\omega \rightarrow 0$ limit of the density response function, we find

$$
\lim _{\omega \rightarrow 0} \operatorname{Im}[\chi(\omega, \vec{k})]=\frac{k^{2} N}{m_{I}[1-R(\alpha)]} \pi \delta\left(\omega^{2}\right),
$$

where we used the following representation for the $\delta$ function:

$$
\delta(x)=\lim _{n \rightarrow 0} \frac{1}{n \pi} \frac{1}{1+(x / n)^{2}} .
$$

So, at temperature zero, the sum rule becomes

$$
\frac{\pi}{2} \frac{k^{2} N}{m_{I}[1-R(\alpha, \vec{k})]}+\int_{\varepsilon}^{\infty} d \omega \omega \operatorname{Im}[\chi(\omega, \vec{k})]=N \frac{\pi}{2} \frac{k^{2}}{m_{I}},
$$

where $\varepsilon$ is a positive infinitesimal. Care has to be taken at finite temperatures since the $\delta$ peak will then broaden and will start to overlap with other contributions of the spectrum. At low enough temperatures, we will see that our results agree well with Eq. (37).

\section{EXTENDED MEMORY FUNCTION FORMALISM}

In Ref. [50], it was noticed that the Feynman polaron model system does not satisfy the sum rule for the density-density correlation. This limitation can be removed by introducing a finite lifetime $\tau$ for the eigenstates of the Feynman model system that mimics scattering events with the bosonic bath, which can be done by replacing $\exp (i \Omega t)$ with $(1+i t / \tau)^{\Omega \tau}$ in Eq. (16). This technique is called the extended memory function formalism and was applied in Ref. [36] for the solid-state Fröhlich polaron. It was shown that, at intermediate coupling, this gives a better agreement between the theory and the diagrammatic quantum Monte Carlo numerical techniques.

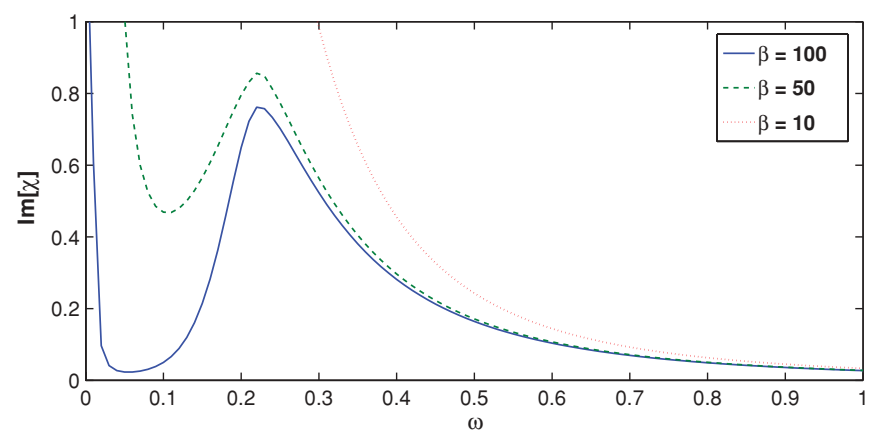

FIG. 2. (Color online) The Bragg response of the polaron system consisting of an impurity in a BEC polaron in the weak-coupling regime $(\alpha=0.1)$ with momentum exchange $k=1$ for different temperatures.
This extension also solves the problem of the inconsistency of the linewidths at strong coupling with the uncertainty relation for the solid-state Fröhlich polaron, which was first mentioned in Ref. [31]. It seems that the second representation for the imaginary part of the memory function (30) is not suitable for the introduction of a lifetime. To obtain a qualitative picture of the dependence of the results on the lifetime, we can work with the real and imaginary parts of the memory function as expressed in Eq. (22).

\section{RESULTS AND DISCUSSION}

The results we obtain in this section are for a system consisting of a sodium condensate with a single lithium impurity, this means that we use, for the bosonic mass, $m_{B}=3.8221$, which is in polaronic units as are all the results in this section.

We begin by looking at the weak-coupling regime. In Fig. 2, the Bragg response (21) is presented as a function of the transferred energy $\hbar \omega$ for different temperatures and for a momentum exchange $k=1$. At low temperatures $(\beta=100)$, we clearly see a peak that represents the weak-coupling scattering process and can be understood as the emission of Bogoliubov excitations. Also, the contribution of the temperature broadened $\delta$ peak at low $\omega$ is seen. This is the anomalous Drude peak (see Ref. [51]). If we look at higher temperatures, the zero-temperature $\delta$ peak broadens, and there is a larger overlap with the weak-coupling scattering peak. At $\beta=10$, this peak has become indistinguishable from the anomalous Drude peak. For $\beta=100$, a distinction between the two contributions can still be made, and the f-sum rule can be applied, as will be done below.

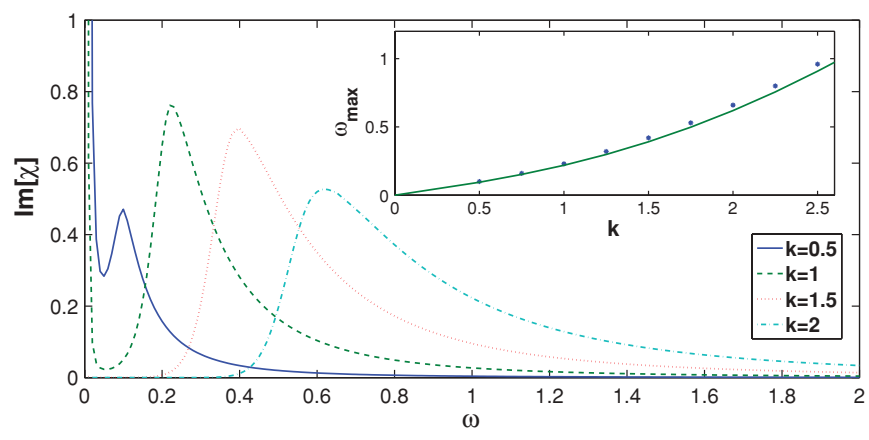

FIG. 3. (Color online) The Bragg response of the polaron system consisting of an impurity in a BEC in the weak-coupling regime ( $\alpha=$ $0.1)$ at temperature $\beta=100$ for different momentum exchanges. The inset shows the frequency of the maximum as a function of the exchanged momentum $k$. The curve gives a least-squares fit of the Bogoliubov spectrum (38), with fitting parameter $m=3.9534$. 

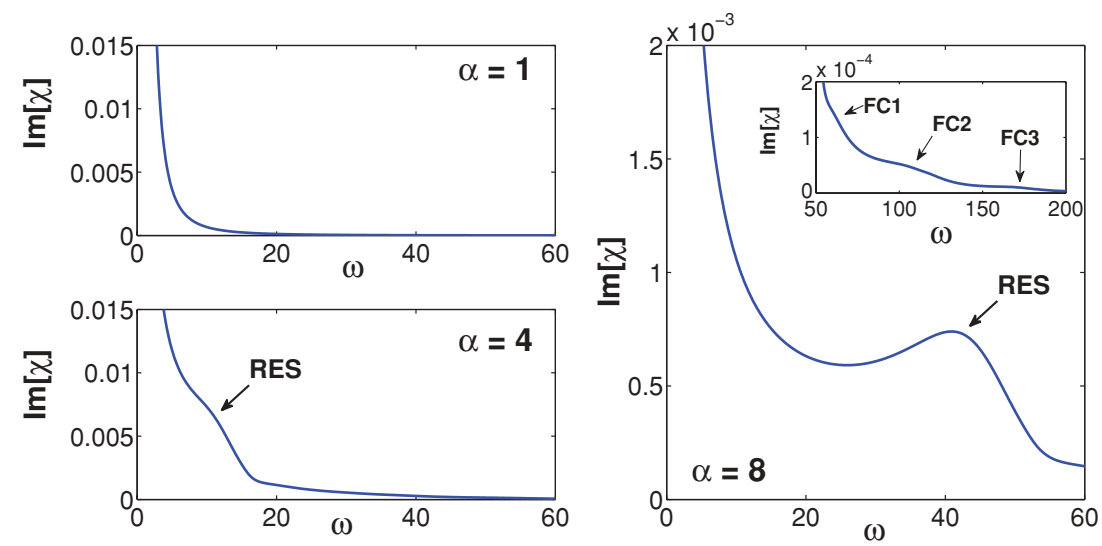

FIG. 4. (Color online) The Bragg response of the polaron system consisting of an impurity in a BEC at different values for the coupling parameter $\alpha$ at temperature $\beta=100$ and a momentum exchange $k=1$. In the strong-coupling regime, the imprint of the RES resonance appears. The inset shows the first three FC peaks at $\alpha=8$.
The dependence of the spectrum on the exchanged momentum $k$ is presented in Fig. 3 for $\alpha=0.1$ and $\beta=100$. For larger momentum exchange, the scattering peak is shifted to higher frequencies, and a broadening is observed. The inset of Fig. 3 shows the frequencies $\omega_{\max }$ at which the maximum of the peak occurs as a function of the exchanged momentum $k$ together with a least-squares fit to the Bogoliubov spectrum,

$$
\omega=\frac{k}{2 m} \sqrt{k^{2}+2},
$$

where $m$ is determined as a fitting parameter to be as follows: $m=3.9534$; which is in good agreement with the bosonic mass of the condensate $\left(m_{B}=3.8221\right)$. This shift, according to the Bogoliubov dispersion, is plausible since the peak corresponds to the emission of Bogoliubov excitations.

In Fig. 4, the high-frequency tail of the Bragg spectrum is shown for different coupling strengths $\alpha$ at $\beta=100$ and $k=1$. In the strong-coupling regime, a resonance is seen that is absent in the weak-coupling regime. This feature is well known from the solid-state Fröhlich polaron and corresponds to a transition to the relaxed excited state (RES); it was first proposed in Ref. [52]. This resonance appears at a frequency $\omega_{\mathrm{RES}}$ such that $\omega_{\mathrm{RES}}^{2}=\operatorname{Re}\left[\Sigma\left(\omega_{\mathrm{RES}}, \vec{k}\right)\right]$ with the supplementary

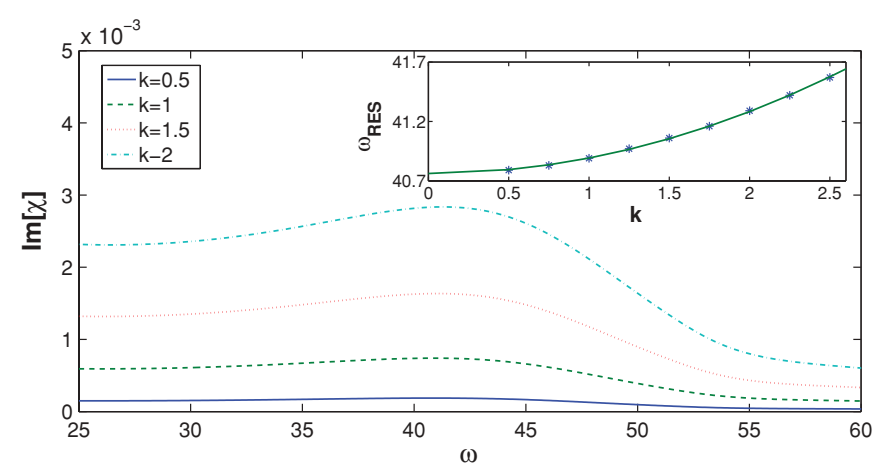

FIG. 5. (Color online) The RES peak in the Bragg response of the polaron system consisting of an impurity in a BEC in the strong-coupling regime $(\alpha=8)$ at temperature $\beta=100$ for different momentum exchanges. The inset shows the frequency of the maximum as a function of the exchanged momentum $k$ at $\beta=100$. The curve is a least-squares fit to the quadratic dispersion (39), with fitting parameters $v=40.76$ and $m^{*}=3.84$. condition $\operatorname{Im}\left[\Sigma\left(\omega_{\mathrm{RES}}, \vec{k}\right)\right] \ll 1$. From Eq. $(21)$, it is clear that these conditions cause a peak in the spectrum. This resonance corresponds to a transition from the polaron ground state to an excited state in the polaronic self-trapping potential that has been relaxed consistent with the new excited wave function of the impurity. The coupling strength where the RES appears in the Bragg spectrum is slightly below $\alpha=4$. This is in agreement with the prediction in Ref. [20] that, for a BEC impurity, the transition between the weak- and the strong-coupling regimes occurs around $\alpha \simeq 3$ for $\beta \rightarrow \infty$. In the strong-coupling regime, other peaks are present that are indicated for $\alpha=8$ in the inset of Fig. 4. These are the FC peaks and represent a transition to the RES together with the emission of Bogoliubov excitations. They only appear in the strong-coupling regime, which was also observed in the case of the acoustic polaron [53].

The dependence of the RES peak on the exchanged momentum is depicted in Fig. 5. The inset shows the frequency of the maximum of the RES peaks as a function of the exchanged momentum $q$ together with a least-squares fit to a quadratic dispersion,

$$
\omega=v+\frac{k^{2}}{2 m^{*}},
$$

where $v$ and $m^{*}$ are the fitting parameters. This suggests that the RES is characterized by a transition frequency $v$ and an effective mass $m^{*}$.

As a consistency test, it was checked whether the above results satisfy the f-sum rule (37). Filling out the expression for the imaginary part of the density response function (21)

TABLE I. Results of a numerical calculation of the left-hand side of expression (40) with a cutoff $\omega_{c}=500$ introduced for the $\omega$ integral.

\begin{tabular}{cccc}
\hline \hline$k$ & $\alpha=1$ & 4 & 8 \\
\hline 1 & 1.5442 & 1.6845 & 1.5532 \\
3 & 1.5482 & 1.6419 & 1.5355 \\
5 & 1.5506 & 1.5555 & 1.4979 \\
\hline \hline
\end{tabular}




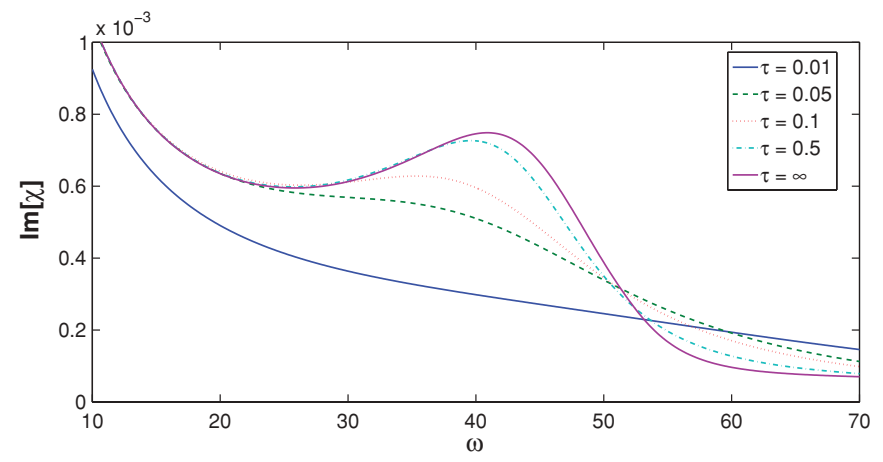

FIG. 6. (Color online) The RES peak in the Bragg response of the polaron system consisting of an impurity in a BEC in the strong-coupling regime $(\alpha=8)$ at temperature $\beta=100$ with different lifetimes $\tau$.

and dividing by the common factor, the f-sum rule takes the form

$$
\begin{aligned}
& \frac{\pi}{2[1-R(\alpha, \vec{k})]}-\int_{\varepsilon}^{\infty} d \omega \omega \\
& \quad \times \frac{\operatorname{Im}[\Sigma(\omega, \vec{k})]}{\left\{\omega^{2}-\operatorname{Re}[\Sigma(\omega, \vec{k})]\right\}^{2}+\{\operatorname{Im}[\Sigma(\omega, \vec{k})]\}^{2}}=\frac{\pi}{2} .
\end{aligned}
$$

It is impossible to integrate numerically to infinity, and for this reason, a cutoff, $\omega_{c}=500$, is used. A calculation of the left-hand side of Eq. (40) results in the values in Table I for different values of $\alpha$ and $k$ at $\beta=100$. These results should be compared with the rigorous value $\pi / 2=1.5708, \ldots$, which gives good agreement with small deviations.

We now give a qualitative analysis of the dependence of the results on the lifetime $\tau$, which was introduced in Sec. VII within the extended memory function formalism. In Fig. 6, the RES peak is presented at different values for $\tau$ in the strongcoupling regime $(\alpha=8)$. It is observed that the inclusion of a lifetime parameter on the order of the polaronic time unit results in a broadening of the peak, where a smaller lifetime corresponds to a broader peak. When a lifetime on the order of a hundredth of the polaronic time unit is introduced, the peak cannot be distinguished anymore.

\section{CONCLUSIONS}

We have derived a general formula for the density response function as a function of the transferred energy and momentum with the Mori-Zwanzig projection operator formalism. This provides a general result that can be used for Bragg spectroscopy but also for other probes that exhibit an arbitrary energy and momentum exchange as, for example, neutron scattering where the output is also determined by the density response function [48]. This is applied to the Fröhlich polaron Hamiltonian for which the well-known results from Ref. [31] for the optical absorption are found. We then extend the analysis to Bragg scattering of impurity polarons in a Bosecondensed gas, where the Bogoliubov excitations play the role of the phonons in the polaron formation. The f-sum rule is checked.

To analyze the results, we introduced the specific system of a lithium impurity in a sodium condensate and calculated the spectra in the different coupling regimes and for different momentum exchanges and temperatures. It is seen that these spectra possess similar features as also found in the optical absorption of the solid-state Fröhlich polaron. Furthermore, it was shown that the weak-coupling scattering peaks follow the Bogoliubov spectrum as a function of the exchanged momentum. In the strong-coupling regime, the RES emerges, and we derive the transition frequency and the effective mass associated with the RES. This is of importance for the comparison with diagrammatic quantum Monte Carlo numerical techniques, which, in the case of the optical absorption of the solid-state Fröhlich polaron, has led to new results concerning the linewidth and oscillator strength of the RES and FC transitions. The FC peaks were also observed.

Our results were tested using the f-sum rule, which resulted in good agreement with small deviations.

The influence of the introduction of a lifetime within the extended memory function formalism was also qualitatively investigated, and it was shown that this results in a broadening of the RES peak.

\section{ACKNOWLEDGMENTS}

This work was supported by FWO-V under Projects No. G.0180.09N, No. G.0115.06, No. G.0356.06, and No. G.0370.09N, and the WOG Belgium under Project No. WO.033.09N. J.T. gratefully acknowledges support of the Special Research Fund of the University of Antwerp, BOF NOI UA 2004. M.O. acknowledges financial support by the ExtreMe Matter Institute EMMI in the framework of the Helmholtz Alliance under Grant No. HA216/EMMI. W.C. acknowledges financial support from the BOF-UA.
[1] F. Brennecke, T. Donner, S. Ritter, T. Bourdel, M. Köhl, and T. Esslinger, Nature (London) 450, 268 (2007).

[2] A. P. Chikkatur, A. Görlitz, D. M. Stamper-Kurn, S. Inouye, S. Gupta, and W. Ketterle, Phys. Rev. Lett. 85, 483 (2000).

[3] A. Öttl, S. Ritter, M. Köhl, and T. Esslinger, Rev. Sci. Instrum. 77, 063118 (2006).

[4] C. Silber, S. Günther, C. Marzok, B. Deh, P. W. Courteille, and C. Zimmermann, Phys. Rev. Lett. 95, 170408 (2005)

[5] G. Modugno, M. Modugno, F. Riboli, G. Roati, and M. Inguscio, Phys. Rev. Lett. 89, 190404 (2002).
[6] Z. Hadzibabic, C. A. Stan, K. Dieckmann, S. Gupta, M. W. Zwierlein, A Görlitz, and W. Ketterle, Phys. Rev. Lett. 88, 160401 (2002).

[7] A. Recati, J. N. Fuchs, C. S. Peça, W. Zwerger, Phys. Rev. A 72, 023616 (2005).

[8] M. J. Bijlsma, B. A. Heringa, and H. T. C. Stoof, Phys. Rev. A 61, 053601 (2000).

[9] G. E. Astrakharchik and L. P. Pitaevskii, Phys. Rev. A 70, 013608 (2004).

[10] M. Girardeau, Phys. Fluids 4, 279 (1961). 
[11] E. P. Gross, Ann. Phys. 19, 234 (1962).

[12] R. M. Kalas and D. Blume, Phys. Rev. A 73, 043608 (2006).

[13] D. K. K. Lee and J. M. F. Gunn, Phys. Rev. B 46, 301 (1992).

[14] C. Mora and F. Chevy, Phys. Rev. A 80, 033607 (2009).

[15] N. Prokof'ev and B. Svistunov, Phys. Rev. B 77, 020408 (2008).

[16] S. Nascimbène, N. Navon, K. J. Jiang, L. Tarruell, M. Teichmann, J. McKeever, F. Chevy, and C. Salomon, Phys. Rev. Lett. 103, 170402 (2009).

[17] A. Schirotzek, C.-H. Wu, A. Sommer, and M. W. Zwierlein, Phys. Rev. Lett. 102, 230402 (2009).

[18] F. M. Cucchietti and E. Timmermans, Phys. Rev. Lett. 96, 210401 (2006).

[19] K. Sacha and E. Timmermans, Phys. Rev. A 73, 063604 (2006).

[20] J. Tempere, W. Casteels, M. K. Oberthaler, S. Knoop, E. Timmermans, and J. T. Devreese, Phys. Rev. B 80, 184504 (2009).

[21] I. Bloch, J. Dalibard, and W. Zwerger, Rev. Mod. Phys. 80, 885 (2008).

[22] T. Best, S. Will, U. Schneider, L. Hackermüller, D. van Oosten, I. Bloch, and D.-S. Lühmann, Phys. Rev. Lett. 102, 030408 (2009).

[23] S. Ospelkaus, C. Opelkaus, O. Wille, M. Succo, P. Ernst, K. Sengstock, and K. Bongs, Phys. Rev. Lett. 96, 180403 (2006).

[24] K. Günter, T. Stöferle, H. Moritz, M. Köhl, and T. Esslinger, Phys. Rev. Lett. 96, 180402 (2006).

[25] M. Bruderer, A. Klein, S. R. Clark, and D. Jaksch, Phys. Rev. A 76, 011605 (2007).

[26] M. Bruderer, A. Klein, S. R. Clark, and D. Jaksch, New J. Phys. 10, 033015 (2008).

[27] A. Privitera and W. Hofstetter, Phys. Rev. A 82, 063614 (2005).

[28] L. D. Landau and S. I. Pekar, Zh. Eksp. Teor. Fiz. 18, 419 (1948).

[29] H. Frölich, Adv. Phys. 3, 325 (1954).

[30] R. P. Feynman, Phys. Rev. 97, 660 (1955).

[31] J. Devreese, J. De Sitter, and M. Goovaerts, Phys. Rev. B 5, 2367 (1972).
[32] R. P. Feynman, R. W. Hellwarth, C. K. Iddings, and P. M. Platzman, Phys. Rev. 127, 1004 (1962).

[33] A. S. Mishchenko, N. Nagaosa, N. V. Prokof'ev, A. Sakamoto, and B. V. Svistunov, Phys. Rev. Lett. 91, 236401 (2003).

[34] J. Tempere and J. T. Devreese, Phys. Rev. B 64, 104504 (2001).

[35] A. S. Alexandrov, Phys. Rev. B 77, 094502 (2008).

[36] G. De Filippis, V. Cataudella, A. S. Mishchenko, C. A. Perroni, and J. T. Devreese, Phys. Rev. Lett. 96, 136405 (2006).

[37] L. Pitaevskii and S. Stringari, Bose-Einstein Condensation (Clarendon Press, Oxford, 2003).

[38] D. M. Stamper-Kurn, A. P. Chikkatur, A. Görlitz, S. Inouye, S. Gupta, D. E. Pritchard, and W. Ketterle, Phys. Rev. Lett. 83, 2876 (1999).

[39] J. Steinhauer, R. Ozeri, N. Katz, and N. Davidson, Phys. Rev. Lett. 88, 120407 (2002).

[40] J. T. Devreese, L. F. Lemmens, and J. Van Royen, Phys. Rev. B 15, 1212 (1977).

[41] J. L. M. van Mechelen, D. van der Marel, C. Grimaldi, A. B. Kuzmenko, N. P. Armitage, N. Reyren, H. Hagemann, and I. I. Mazin, Phys. Rev. Lett. 100, 226403 (2008).

[42] J. T. Devreese, S. N. Klimin, J. L. M. van Mechelen, and D. van der Marel, Phys. Rev. B 81, 125119 (2010).

[43] H. Mori, Prog. Theor. Phys. 33, 423 (1965).

[44] R. Zwanzig, Phys. Rev. 124, 983 (1961).

[45] F. M. Peeters and J. T. Devreese, Phys. Rev. B 28, 6051 (1983).

[46] J. T. Devreese, e-print arXiv:1012.4576v1.

[47] M. Ichiyanagi, J. Phys. Soc. Jpn. 32, 604 (1972).

[48] L. Van Hove, Phys. Rev. 95, 249 (1954).

[49] G. D. Mahan, Many-Particle Physics, 2nd ed. (Plenum, New York, 1990).

[50] V. Cataudella, G. Filippis, and C. A. Perroni, Polarons in Advanced Materials (Springer, Netherlands, 2008).

[51] J. T. Devreese and J. Tempere, Solid State Commun. 106, 309 (1998).

[52] E. Kartheuser, R. Evrard, and J. Devreese, Phys. Rev. Lett. 22, 94 (1969).

[53] F. M. Peeters and J. T. Devreese, Phys. Rev. B 35, 3745 (1987). 